\title{
Desenvolvimento corporal e relação entre biometria e peso de cordeiros lactantes da raça Santa Inês criados na Amazônia
}

\author{
[Growth of Santa Inês lambs until weaning and relationship between biometric measurements \\ and body weight, when raised in the Amazonia climate] \\ D.S. Souza ${ }^{1}$, H.P. Silva ${ }^{1}$, J.M.P. Carvalho ${ }^{1}$, W.O. Melo $^{2 *}$, B.M. Monteiro ${ }^{3}$, D.R. Oliveira ${ }^{1}$ \\ ${ }^{1}$ Universidade Luterana do Brasil - ULBRA - Santarém, PA \\ ${ }^{2}$ Universidade Federal Rural da Amazônia - UFRA - Paragominas, PA \\ ${ }^{3}$ Universidade de São Paulo - USP - São Paulo, SP
}

\begin{abstract}
RESUMO
Objetivou-se com este trabalho descrever as medidas biométricas de cordeiros lactantes da raça Santa Inês criados em condições amazônicas; estabelecer relação entre a idade e as medidas corporais; bem como correlacionar essas medidas biométricas com o peso corporal. Utilizaram-se 75 animais criados em sistema semi-intensivo, submetidos a mensurações do peso corporal (PC), comprimento corporal (CC), altura do anterior (AA) e do posterior (AP), perímetro torácico (PT), largura da garupa (LG) e do peito (LP), comprimento da perna (CP), perímetro da perna (PP) e compacidade corporal (COMPC), ao nascer e aos 15, 30, 45 e 60 dias de idade. Somente as medidas LG e PP demonstraram regressões com $\mathrm{R}^{2}$ abaixo de 0,70 em relação à idade dos cordeiros. Todas as medidas corporais utilizadas nesta pesquisa, individualmente, demonstraram $r$ positivo com o peso corporal. As variáveis biométricas que melhor compuseram a equação de predição do peso dos cordeiros utilizados neste estudo foram CC, AP, PT, LG e PP. Concluiu-se que a idade de cordeiros pode ser utilizada como preditora do peso e de algumas medidas biométricas corporais e que o peso pode ser estimado por meio de algumas medidas biométricas.
\end{abstract}

Palavras-chave: ovinocultura, produtividade, medidas corporais, predição

\begin{abstract}
This study aimed to 1) describe the biometric measurements of Santa Inês lambs until weaning, when raised in the Amazonia conditions; 2) establish the relationship between age and body measurements; and 3) correlate these biometric measurements to body weight. 75 animals submitted to semi-intensive breeding were used, and underwent measurements of body weight $(B W)$, body length $(B L)$, height of the forelimb $(\mathrm{HF})$ and hindlimb $(\mathrm{HH})$, thoracic perimeter (TP), width of rump (WR) and chest (WC), length of leg (LL), perimeter of leg (PL) and more body compacity (COMP), at birth and at 15, 30, 45 and 60 days of age. As a result, the WR and PL measurements demonstrated regressions with $R^{2}$ below 0.70 in relation to age of lambs. All biometrical measurements used in this study individually demonstrated positive $r$ with body weight. Biometric variables that compose the equation for predicting weight of lambs used in this study were BL, HH, TP, WR and PL. It was concluded that the age of lambs can be used as a predictor of weight and some body biometric measurements. The weight can also be estimated by some biometric measurements.
\end{abstract}

Keywords: sheep, productivity, body measurements, prediction

\section{INTRODUÇÃO}

A ovinocultura brasileira é uma atividade econômica promissora, uma vez que há demanda de carne dessa espécie no mercado interno e crescimento no consumo (Vargas Junior et al.,

Recebido em 12 de fevereiro de 2014

Aceito em 21 de agosto de 2014

*Autor para correspondência (corresponding author)

waldjaniomelo@zootecnista.com.br
2011). O efetivo rebanho de ovinos tem crescido, alcançando 17,3 milhões de cabeças em 2010, obtendo aumento de 3,4\% em relação a 2009. No estado do Pará, essa atividade está em crescente desenvolvimento, com um rebanho efetivo de 203.608 mil cabeças, que corresponde a 35\% do total da região Norte (IBGE, 2011). 
Dentre as raças de ovinos criadas no Brasil, a Santa Inês vem sendo amplamente utilizada para produção de carne, por ser adaptada à maioria das regiões brasileiras e apresentar bons resultados de produção e qualidade da carne (Pinheiro e Jorge, 2010).

Dentre as ferramentas utilizadas para mensurar a produtividade de pequenos ruminantes, tem-se destacado a biometria corporal, que, quando analisada juntamente com outros índices zootécnicos, constitui uma importante base de dados para a avaliação individual dos animais e para determinar a evolução do sistema produtivo (Yáñez et al., 2004). As medidas biométricas obtidas de animais in vivo apresentam alta correlação com as medidas da carcaça e podem ser utilizadas em conjunto, ou isoladamente, para estimar as medidas da futura carcaça (Cunha et al., 1999; Rosa, 1999), além de poderem ser empregadas para indicar a proporção de músculos e tecido adiposo, orientando os produtores quanto ao melhor momento de abate dos animais (Pinheiro et al., 2007; Moreno, 2010).

Em estudo recente, Koritiaki et al. (2012) mostraram que as medidas corporais estão correlacionadas positivamente com o peso, e as regressões para estimar o peso por meio das medidas foram eficientes, principalmente as regressões lineares múltiplas compostas por mais de uma medida corporal. Dessa forma, mostra-se que é possível predizer o peso corporal devido à alta correlação existente entre as medidas de biometria corpórea e o peso vivo (Wood e MacFie, 1980; Souza et al., 2009; Koritiaki et al., 2012).

Animais criados com variações climáticas fora da faixa de conforto térmico podem apresentar perda de peso, crescimento retardado, problemas respiratórios e hormonais, falta de apetite e baixo rendimento de carcaça (Oliveira, 2011), indicando assim a necessidade de realizar pesquisas que visam avaliar o crescimento e desenvolvimento ponderal de cordeiros em condições de climas diferentes. Nesse contexto, é necessário que o fator climático seja levado em consideração, pois as condições amazônicas apresentam-se como estressantes devido às altas temperaturas e também umidade relativa do ar (Costa et al., 2009).
Apesar de algumas pesquisas serem conduzidas para verificar a biometria de cordeiros e sua correlação com o peso vivo (Cunha Filho et al., 2010; Jucá et al., 2011; Castro et al., 2012) em diversas regiões brasileiras, ainda não houve estudos na região Amazônica analisando esses parâmetros, indicando a necessidade desses dados para o desenvolvimento da ovinocultura. Dessa forma, este trabalho teve como objetivos: 1) descrever as medidas biométricas de cordeiros lactantes da raça Santa Inês criados em condições amazônicas; 2) estabelecer relação entre a idade e as medidas corporais de cordeiros lactantes da raça Santa Inês; bem como 3) correlacionar essas medidas biométricas com o peso corporal desses cordeiros.

\section{MATERIAL E MÉTODOS}

O estudo foi conduzido em três fazendas localizadas no município de Santarém, na mesorregião do Baixo Amazonas, no estado do Pará, no período de agosto a novembro de 2011. O clima da região é caracterizado como quente e úmido, do tipo $\mathrm{AW}$, de acordo com a classificação de Kõppen, com pluviometria anual média de $1.920 \mathrm{~mm}$, com maior intensidade entre os meses de dezembro e maio (Sudan, 1984).

Para a realização desta pesquisa, foram utilizados 75 cordeiros machos lactantes da raça Santa Inês. Todos os cordeiros selecionados para a pesquisa tinham alto padrão genético e eram clinicamente sadios. Após o nascimento, os animais foram mantidos em aleitamento ininterrupto com suas mães até o momento do desmame, aos 60 dias de vida. O lote de matrizes e cordeiros foi mantido sob sistema semi-intensivo de criação, com pastos formados por Brachiaria brizantha e $B$. humidicola, além de fornecimento ad libitum de mistura mineral específica para ovinos, mais 150 gramas de concentrado por dia (suplemento à base de $30 \%$ de farelo de soja e $70 \%$ de milho triturado) somente para as mães.

Todos os cordeiros foram submetidos a mensurações do peso corporal (kg), comprimento corporal $(\mathrm{cm})$, altura do anterior e do posterior $(\mathrm{cm})$, perímetro torácico $(\mathrm{cm})$, largura da garupa e do peito $(\mathrm{cm})$, comprimento da perna $(\mathrm{cm})$, perímetro da perna $(\mathrm{cm})$ e compacidade corporal, ao nascer e aos 15, 30, 45 e 60 dias de idade. 
A pesagem corporal foi realizada individualmente em balança própria para ovinos jovens. A morfometria corporal dos cordeiros foi obtida com o auxílio de fita métrica, com o animal mantido em estação, com posição de aprumos, conforme metodologia sugerida por Yañez et al. (2004).

O comprimento corporal (CC) foi obtido pela distância entre a articulação cervicotorácica e a base da cauda na primeira articulação intercoccígea. A altura do anterior foi medida entre a distância da região da cernelha e a extremidade distal do membro anterior. A altura do posterior foi mensurada pela distância entre a tuberosidade sacra e a extremidade distal do membro posterior.

A medida do perímetro torácico foi obtida tomando-se como base a circunferência externa da cavidade torácica, junto às axilas. Para a largura da garupa foi considerada a distância entre os trocânteres maiores dos fêmures. A largura do peito foi obtida pela distância entre as faces laterais das articulações escapuloumerais.

O comprimento da perna foi mensurado pela distância entre o trocânter maior do fêmur e o bordo da articulação tarsometatarsiana. O perímetro da perna foi obtido tomando como base a parte média da perna, acima da articulação femorotibiorrotuliana.

Foi estimada também a compacidade corporal (COMPAC), índice objetivo da conformação in vivo, obtido pela fórmula COMPAC= peso vivo (PV) / comprimento corporal (CC) $(\mathrm{kg} / \mathrm{cm})$, sendo que, quanto maior a compacidade corporal, maior a proporção de músculos e gordura no animal.

A análise descritiva dos dados, representada pelas médias e os coeficientes de variação (CV), foi obtida pelo programa SAS versão 9.2 (SAS/STAT, SAS Institute Inc., Cary, NC), procedimento MEANS. As equações de predição da idade (dias) dos cordeiros foram calculadas utilizando-se o peso e as medidas corporais em relação aos momentos de idade (polinômios: nascimento, 15, 30, 45, e 60 dias) pelo procedimento GLM do SAS, por meio da metodologia dos quadrados mínimos para regressão simples polinomial.

Foi avaliada a correlação entre o peso corporal e as demais variáveis de medidas biométricas corporais, pelo procedimento CORR do SAS. Devido à multicolinearidade entre as medidas estudadas, também foi realizada a determinação de uma equação que melhor pudesse predizer o peso corporal dos cordeiros utilizando as medidas biométricas corporais. Para tanto, foi realizada regressão linear múltipla pelo procedimento REG do SAS, usando o modelo STEPWISE.

Para as regressões e correlações calculadas, foram considerados altos os coeficientes de determinação $\left(R^{2}\right) \geq 0,70$ e coeficientes de correlação $(r) \geq 0,50$. O valor de $p$ foi considerado significativo quando $<0,05$. As figuras foram confeccionadas por meio do programa SigmaPlot, versão 10.0.

\section{RESULTADOS E DISCUSSÃO}

Em linhas gerais, pôde-se observar que o peso e quase todas as medidas corporais mensuradas nesta pesquisa puderam ser preditas por meio da idade dos cordeiros utilizados. Somente as medidas de largura de garupa e perímetro de perna demonstraram regressões polinomiais com coeficiente de determinação abaixo de 0,70 (Tab. 1). Todas as medidas corporais utilizadas nesta pesquisa, individualmente, demonstraram coeficiente de correlação positivo com o peso corporal (Tab. 2). Por fim, as variáveis biométricas que melhor compuseram a equação de predição do peso dos cordeiros utilizados neste estudo foram o comprimento corporal, altura de posterior, perímetro torácico, largura de garupa e perímetro de perna (Tab. 3).

Estudo conduzido por Castro et al. (2012) mostrou valores semelhantes na altura do

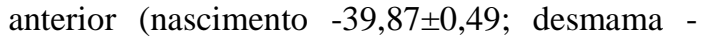
54,78 $\pm 0,91$;) em cordeiros da raça Santa Inês criados no município de Londrina-PR, e inferiores em relação ao perímetro torácico e peso corporal tanto no nascimento $(37,59 \pm 0,63 \mathrm{e}$ $3,82 \pm 015)$ como no desmame aos 70 dias $(56,14 \pm 0,79$ e $54,78 \pm 0,91)$. 
Tabela 1. Desenvolvimento das medidas de biometria corporal de cordeiros lactantes da raça Santa Inês e suas respectivas regressões polinomiais

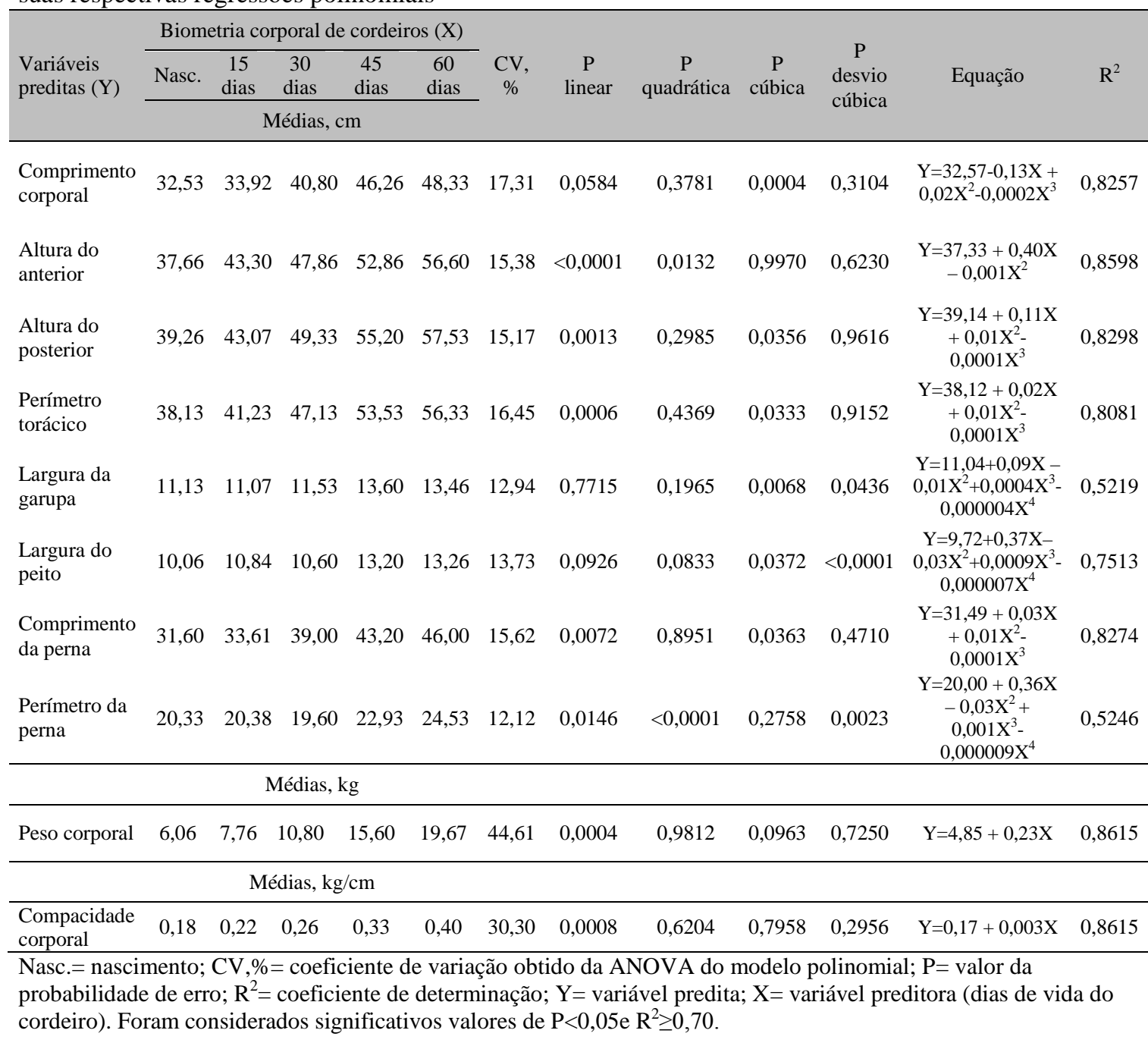

No entanto, Jucá et al. (2011) encontraram resultados superiores ao deste trabalho, tendo em vista Largura da Garupa (LG de 0,19 ao nascer e 0,25 no desmame), Largura de Peito (LP de 0,23 ao nascer e 0,60 no desmame) e Perímetro de Perna (PP de 0,08 ao nascer e $0,31 \mathrm{~cm}$ ao desmame). Vale considerar que esses dados foram coletados de animais ao nascer até 120 dias de idade.

No presente trabalho, o maior coeficiente de variação nos dados foi observado para o peso corporal (44,61\%), indicando ser uma característica mais sujeita às variáveis externas, concordando com os resultados obtidos por Cunha Filho et al. (2010). Mesmo assim, o peso ao nascimento $(6,06 \mathrm{~kg})$ e ao desmame $(19,67 \mathrm{~kg})$, obtidos na presente pesquisa, foram superiores aos compilados por Pereira et al.(2008) em ovinos criados nos estados de Rondônia (2,78 e $13,44 \mathrm{~kg})$, Acre (2,90 e 13, 50kg) e Pará (3,23 e $13,50 \mathrm{~kg})$ e aos encontrados nos estados de Goiás (4,27 e 17, 20kg) (Oliveira, 2011) e Paraná (3,69 e 12,47kg) (Castro et al., 2012).

Em condições climáticas amazônicas, Oliveira et al. (2013) verificaram peso de cordeiros da raça Santa Inês de $3,73 \mathrm{~kg}$ ao nascimento e $14,82 \mathrm{~kg}$ ao desmame, quando submetidos a amamentação controlada e sendo amamentados por ovelhas criadas em sistema de criação semi-intensivo. Esses pesos se mantiveram abaixo dos encontrados no presente estudo. Apesar das semelhanças entre o clima do ambiente criatório 
e o manejo nutricional a que as mães dos cordeiros foram submetidas, não foi possível comparar os reais efeitos genéticos e do sexo dos cordeiros, visto que Oliveira et al. (2013) provavelmente utilizaram cordeiros machos e fêmeas, diferentemente desta pesquisa, que utilizou somente machos.

A compacidade corporal é um índice que estima objetivamente a conformação dos animais vivos quanto ao acúmulo de músculos na carcaça, a partir de dois valores de fácil determinação, peso vivo e comprimento corporal. Neste estudo, foi encontrado valor médio de $0,28 \mathrm{~kg} / \mathrm{cm}$, próximo aos dados encontrados por Silva et al. (2012) que, pesquisando ovinos deslanados, obtiveram 0,35 e $0,26 \mathrm{~kg} / \mathrm{cm}$.

\section{(A)}

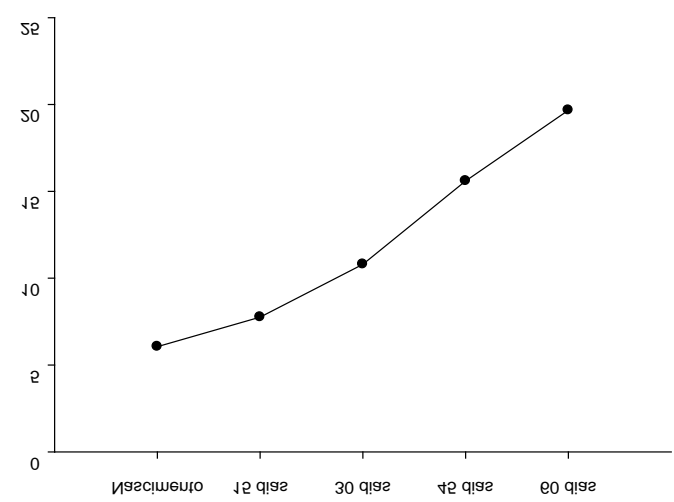

(B)

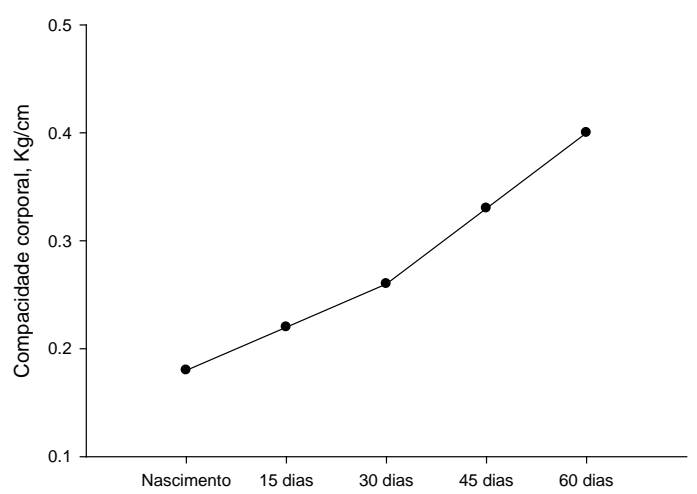

Foram observados altos $\mathrm{R}^{2}$ para a maioria das regressões polinomiais em relação aos cinco momentos da idade dos animais, observando valores $\geq 0,70$. Somente para duas variáveis Perímetro de Perna (PP) e Largura da Garupa (LG) - foram observados valores intermediários de $R^{2}$ (PP - 0,5246; e LG - 0,5219). Analisando as curvas das variáveis (Fig. 1) e os altos coeficientes de determinação (Tab. 1), pode-se afirmar que o desenvolvimento corporal dos cordeiros foi crescente, de acordo com os momentos (nascimento, 15, 30, 45 e 60 dias) de vida dos mesmos.
(C)

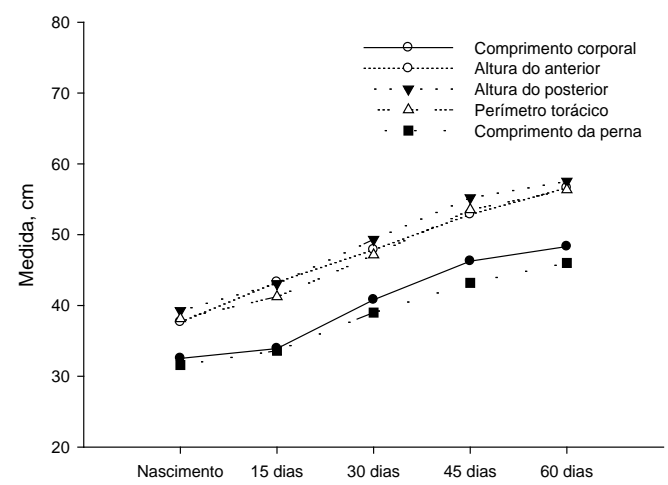

(D)

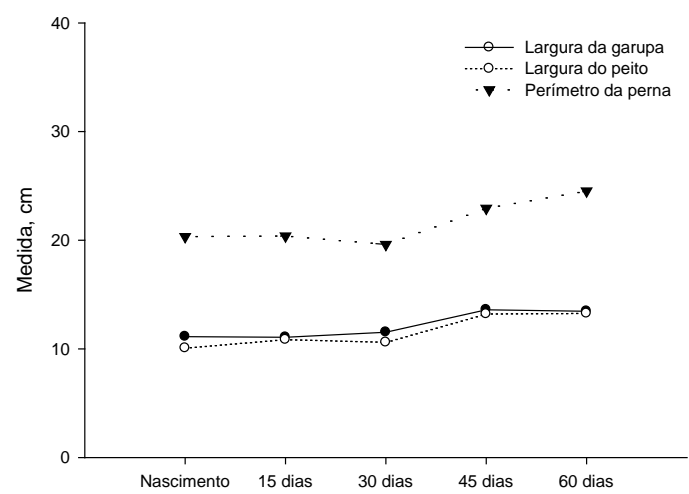

Figura 1. Desenvolvimento das medidas corporais de cordeiros lactentes da raça Santa Inês. (A) Peso corporal; (B) Compacidade corporal; (C) Comprimento corporal, altura do anterior, altura do posterior, perímetro torácico e comprimento da perna; (D) Largura da garupa, largura do peito e perímetro da perna. 
De acordo com estudo realizado sobre a correlação entre o peso corporal e as medidas biométricas de cordeiros (Tab. 2), observou-se que o perímetro torácico, a altura do posterior, a altura do anterior, o comprimento corporal, o comprimento de perna, a largura de peito e a largura da garupa obtiveram valores acima do coeficiente de correlação considerado satisfatório $(r \geq 0,50)$. Somente o perímetro de perna obteve valor marginal ao estipulado, o que também foi observado em outras pesquisas (Gusmão et al., 2009; Vargas Junior et al., 2011).

Tabela 2. Coeficientes de correlação $(r)$ e significância $(p)$ entre o peso corporal e as medidas biométricas de cordeiros

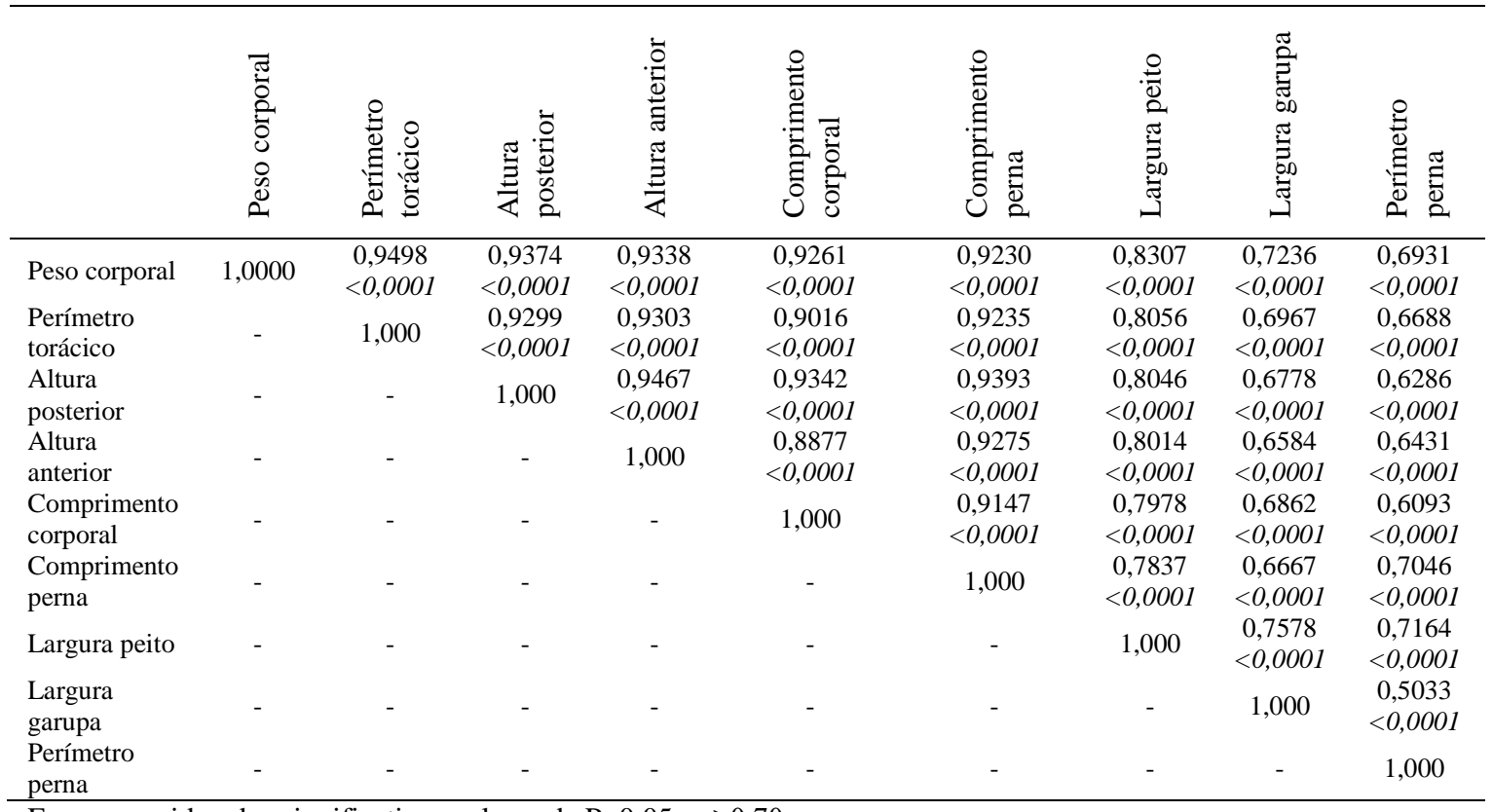

Foram considerados significativos valores de $\mathrm{P}<0,05 \mathrm{e} r \geq 0,70$.

Apesar de o perímetro de perna ter apresentado correlação positiva e significativa com o peso corporal (Tab. 2), Souza et al. (2009) registram que essa é uma medida que apresenta complicações ao ser mensurada, pois se trata de uma região que envolve muitos tecidos musculares, podendo, assim, uma contração ou um relaxamento interferir no resultado, o que nos leva a descartar tal parâmetro para seleção.

Embora as medidas de largura de garupa e largura de peito tenham apresentado significância igualmente elevada (Tab. 2), os valores de $r$ foram menores que os das outras medidas. Portanto, isoladamente, essas medidas seriam menos indicadas para estimar o peso corporal dos animais. Resultados semelhantes também foram obtidos por Souza et al. (2009) em animais de diferentes grupos genéticos.

O parâmetro que apresentou maior $r$ com o peso vivo foi o perímetro torácico $(0,9408 ; p<0,0001)$. Apesar da alta correlação dessa variável com o peso vivo, todas as medidas biométricas analisadas foram altamente significativas, e com alto $r$, corroborando os resultados obtidos por Vargas Junior et al.(2011) e Castro et al. (2012) em cordeiros pantaneiros e Santa Inês, respectivamente. Além disso, os coeficientes de correlação observados nesta pesquisa foram superiores aos encontrados por Castro et al. (2012) no nascimento $(0,68)$ e desmame $(0,86)$, e Cunha Filho et al. (2010) em cordeiros da raça Texel $(0,85)$.

Com base nas variáveis biométricas mensuradas e seus respectivos graus de correlação com o peso vivo, foi possível estimar uma equação linear múltipla que predissesse o peso vivo dos cordeiros estudados (Tab. 3). Observou-se que o $\mathrm{R}^{2}$ obtido nessa equação teve valor superior ao proposto por este estudo $(\geq 0,70)$. Baseando-se nessa regressão linear múltipla, foi possível estimar o peso vivo dos cordeiros da raça Santa Inês por meio das medidas corporais. 
Desenvolvimento corporal...

Tabela 3. Equação de predição do peso corporal por meio das medidas biométricas de comprimento corporal, altura do posterior, perímetro torácico, largura da garupa e perímetro da perna

\begin{tabular}{|c|c|c|c|}
\hline $\begin{array}{l}\text { Variável } \\
\text { predita }\end{array}$ & Equação preditora & $\begin{array}{c}\mathrm{R}^{2} \\
\text { ajustado }\end{array}$ & $\mathrm{P}$ \\
\hline $\begin{array}{c}\text { Peso } \\
\text { corporal }\end{array}$ & $\begin{array}{c}\text { Peso Corporal }=-23,78+0,18(\text { comprimento corporal })+0,14 \text { (altura } \\
\text { posterior) } \\
+0,29 \text { (perímetro torácico) }+0,25(\text { largura garupa })+0,19 \text { (perímetro } \\
\text { perna })\end{array}$ & 0,9358 & $<0,0001$ \\
\hline
\end{tabular}

$\mathrm{P}=$ valor da probabilidade de erro; $\mathrm{R}^{2}=$ coeficiente de determinação. Foram considerados significativos valores de $\mathrm{P}<0,05$ e $\mathrm{R}^{2} \geq 0,70$.

Apesar de encontrarem coeficientes de determinação menores que o presente estudo em várias equações lineares múltiplas para predição do peso vivo $\left(\mathrm{R}^{2} \leq 0,80\right)$, Koritiaki et al. (2012) verificaram que as regressões lineares múltiplas quase sempre apresentaram melhores coeficientes de determinação que as regressões lineares simples, indicando que equações utilizando mais de uma mensuração corporal são mais eficientes para estimar o peso vivo que equações utilizando somente uma mensuração.

\section{CONCLUSÕES}

Foi possível concluir que: 1. A idade de cordeiros lactantes entre o nascimento e 60 dias de idade pode, individualmente, ser utilizada como preditora do peso e de todas as medidas biométricas corporais, com exceção das medidas de largura de garupa e perímetro de perna. 2. Nessas condições criatórias, o melhor modelo para predizer o peso vivo de cordeiros lactantes até os 60 dias de vida foi o composto pelas variáveis comprimento corporal, altura posterior, perímetro torácico, largura de garupa e perímetro de perna.

\section{REFERÊNCIAS}

CASTRO, F.A.B.; RIBEIRO, E.L.A.; KORITIAKI, N.A. et al. Desempenho de cordeiros Santa Inês do nascimento ao desmame filhos de ovelhas alimentadas com diferentes níveis de energia. Semina: Cienc. Agrar., v.33, p.3379-3388, 2012.

COSTA, E.P.S.; TAKEDA, F.R.P.C.; LIMA, R.S. Adaptabilidade de ovinos da raça Santa Inês ao clima amazônico. In CONGRESSO BRASILEIRO DE ZOOTECNIA, 19., 2009, Águas de Lindoia. Anais... Águas de Lindoia: Associação Brasileira de Zootecnia, 2009. p.1-4. (Resumo).
CUNHA, E.A.; BUENO, M.S.; SANTOS, L.E. Correlações entre características de carcaça de cordeiros Suffolk. In: REUNIÃO ANUAL DA SOCIEDADE BRASILEIRA DE ZOOTECNIA, 36., 1999, Porto Alegre. Anais... Porto Alegre: Sociedade Brasileira de Zootecnia, 1999. (Resumo).

CUNHA FILHO, L.F.C.; REGO, F.C.A.; BARCA JUNIOR, F.A. et al. Predição do peso corporal a partir de mensurações corporais em ovinos Texel. Arq. Cienc. Vet. Zool. UNIPAR., v.13, p.5-7, 2010.

GUSMÃO FILHO, J.D.; TEODORO, S.M.; CHAVES, M.A.; OLIVEIRA, S.S. Análise fatorial de medidas morfométricas em ovinos tipo Santa Inês. Arch. Zootec., v. 58, p. 1-4, 2009.

IBGE - INSTITUTO BRASILEIRO DE GEOGRAFIA E ESTATÍSTICA. Levantamento sistemático da produção agrícola. Rio de Janeiro, v. 24, p.1-82, 2011.

JUCÁ, A.F.; PINTO, L.F.B.; MOITA, A.K.F. et al. Correlações entre medidas morfométricas e peso vivo em ovinos Santa Inês a desmama. In: CONGRESSO BRASILEIRO DE ZOOTECNIA, 21., 2011, Maceió. Anais... Maceió: Sociedade Brasileira de Zootecnia, 2011.p.1-3. (Resumo).

KORITIAKI, N.A.; RIBEIRO, E.L.A.; FERNANDES JUNIOR, F. et al. Predição do peso vivo a partir de mensurações corporais em cordeiros Santa Inês. Rev. Syn. Scy UTFPR., v.7, p. 1-3, 2012.

MORENO, G.M.B.; SILVA SOBRINHO, A.G.; LEÃO, A.G. et al. Características morfológicas "in vivo" e da carcaça de cordeiros terminados em confinamento e suas correlações. Rev. Bras. Saúde Prod. Anim., v.11, p.888-902, 2010.

OLIVEIRA, E.M. Tolerância ao calor, medidas morfometricas e cortes comerciais em diferentes grupos genéticos de ovinos. 2011. 108f. Tese (Doutorado em Ciências Animais) - Faculdade de Agronomia e Medicina Veterinária, Universidade de Brasília, Brasília. 
OLIVEIRA, M.E.F.; SOUSA, H.L.L.; MOURA, A.C.B. et al. The effects of parturition season and suckling mode on the puerperium of Santa Ines ewes and on the weight gain of lambs. Arq. Bras. Med. Vet. Zootec., v.65, p.857-864, 2013.

PEREIRA, R.G.A.; COSTA, N.L.; TOWNSEND, C.R.; MAGALHÃES, J.A. Ovinos como componente dos sistemas produtivos amazônicos. Comunicado técnico 337, Embrapa Rondônia, Porto Velho-RO, 2008.

PINHEIRO, R.S.B.; JORGE, A.M. Medidas biométricas obtidas in vivo e na carcaça de ovelhas de descarte em diferentes estágios fisiológicos. Rev. Bras. Zootec., v.39, p.440-445, 2010.

PINHEIRO, R.S.B.; SILVA SOBRINHO, A.G.; MARQUES, C.A.T.; YAMAMOTO, E S.M. Biometría in vivo e da carcaça de cordeiros confinados. Arch. Zootec., v.56, p.955-958, 2007.

ROSA, A.N. Variabilidade fenotípica e genética do peso adulto e da produtividade acumulada de matrizes em rebanhos de seleção da raça Nelore no Brasil. 1999. 114f. Tese (Doutorado) - Faculdade de Medicina de Ribeirão Preto, Universidade de São Paulo, Ribeirão Preto.
SILVA, N.V.; COSTA, R.G.; MEDEIROS, A.N. et al. Efeito do feno de flor-de-seda sobre a carcaça e constituintes corporais de cordeiros Morada Nova, Arch. Zootec., v.61, p.63-70, 2012.

SOUZA, S.; LEAL, A.; BARIONI, C.; MATOS, A. Utilização de medidas biométricas para estimar peso vivo em ovinos. Arch. Latinoam. Prod. Anim, v.17, p.61-66, 2009.

SUDAN. Zoneamento agroecológico do município de Alenquer, Estado do Pará. 1984.

VARGAS JUNIOR, F.M.; MARTINS, C.F.; SOUZA, C.C. et al. Avaliação Biométrica de Cordeiros Pantaneiros. Dourados, v.4, p.60-65, 2011.

WOOD, J.D.; MACFIE, H.J.H. The significance of breed in the prediction of lamb carcass composition from fat thickness measurements. Anim. Prod. Sci., v.31, p.315-319, 1980.

YÁÑEZ, E.A.; RESENDE, K.T.; FERREIRA, A.C.D. et al. Utilização de Medidas Biométricas para Predizer Características Cabritos Saanen. Rev. Bras. Zootec., v.33, p.1564-1572, 2004 